\title{
DYNAMIC PRICING OF ELECTRICITY IN RETAIL MARKETS
}

\author{
Chefi TRIKI \\ Department of Mathematics \\ University of Lecce \\ Via per Arnesano 73100 Lecce - Italy \\ e-mail: chefi.triki@unile.it \\ Antonio VIOLI \\ CESIC-NEC \\ c/o University of Calabria \\ Arcavacata 87036 Rende (CS) - Italy \\ e-mail: antonio.violi@cesic.neceur.com
}

\begin{abstract}
This paper aims at defining a dynamic and flexible tariff structure for a distribution company that protects the retail consumers against the excessive fluctuations of the wholesales market prices. We propose a two-stage pricing scheme that sets in a first-stage a time-of-use tariff that is corrected later by a dynamic component once the real-time demand has been observed. A personalized tariff scheme may be offered by a distribution company to each dynamic customer by allowing him to choose the appropriate robustness level expressed in terms of variability between the first- and the second-stage decisions. The arising limited recourse model has been tested on realistic test problems, by using a slight modification of a recently proposed interior point solution framework.
\end{abstract}

Keywords: Electricity market, Pricing methods, Stochastic Programming

MSC code: $90 \mathrm{C} 15$

\section{Introduction}

The liberalization of electricity markets has become a worldwide concern, involving many countries. As a result, many challenges are being generated and consequently new optimisation models are being developed since most of the old models used by the monopolistic utilities are not useful anymore. However, since the first deregulation of the electricity market in England, a lot of interest has been devoted to the enhancement of competition from the generation side ignoring the potential role that the retail market can have to ensure the efficiency of the restructured markets. More specifically, regulators and distribution companies have always assumed retail demand as inelastic and thus unable to influence the market clearing price or to prevent against market power. This assumption can damage the success of competitive markets especially in case of generation shortage or entry barrier for suppliers.

The most significant signal of alarm received in this direction arrived in 2000 with the crisis of the Californian electricity market when the wholesale prices increased remarkably 
and retail revenues were not sufficient to cover the distribution companies' expenses. Several analysis, sometimes contradictory, have been given for the causes of that crisis but it seems that most of the researchers agreed on at least one point: the lack of demand responsiveness mechanisms in the retail market was one of the main factors that had contributed in the failure of the Californian experience (see Faruqui and George 2002, Wolak 2001, Borenstein 2004).

Since that time, many works have focused on the techniques that can force the demand to be responsive to wholesale prices. It is widely accepted that real-time pricing for the retail market is an efficient tool to achieve demand elasticity in the electricity systems (Wolak 2001, Borenstein and Holland 2003). The idea is to avoid static tariffs in the retail market and offer new schemes based on the effective energy cost which follows the wholesale prices. This objective, however, is often contrasted with the view of the regulators whose mission is to protect retail customers against the volatility risk and who prefer traditional tariffs such as flat-rates, time-of-use or critical peak pricing (Doucet and Kleit 2003). Reaching a compromise by defining dynamic tariffs that do not expose retail customers to prices volatility seems to be a real challenge towards the completion of the deregulation process in the electricity business.

This paper wants to be a contribution in this direction. It deals with the use of the twostage stochastic programming framework to formulate a dynamic pricing scheme for the retail market. The main idea is the following: the customer is offered a first-stage static tariff that is then followed by a recourse dynamic price once his effective demand is observed. The volatility risk is hedged by limiting the variability between the static and the dynamic components of the tariff. According to this strategy, the dynamic pricing process can be schematised as shown in Figure 1.

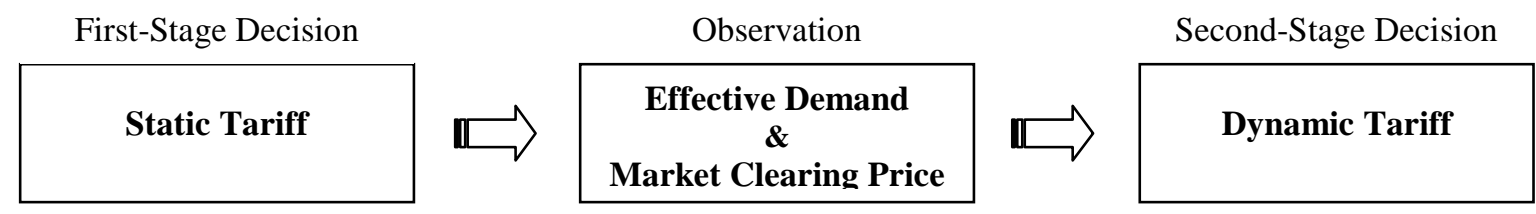

Figure 1: Sequence of events in the two-stage decision process

The stochastic demand is represented explicitly as a random variable with a known probability distribution and is incorporated into the model by means of a set of $L$ scenarios. To each scenario $l$ (with $l=1, \ldots, L$ ) is associated a probability value $p_{l}$ such that $\sum_{l=1}^{L} p_{l}=1$.

The literature on this topic is quite rich. Many authors (most of them are economists) have discussed the importance of real-time retail pricing and some of its effects, for example, on the investment efficiency (Borenstein and Holland 2003) and on the long-term operation (Borenstein 2004). However, to the best of our knowledge only very few tariff definition models based on real-time pricing have been proposed. For example, Petterssen 2004 describes in his thesis a model of a domestic demand-side response and proposes a game theory approach to the strategic interaction between a retailer and an "active" consumer. Another contribution is due to the EFFLOCOM project in which the researchers have developed, tested and evaluated a set of economical incentives whose aim is to improve flexibility in the consumption-side.

The optimisation model we are proposing here should be considered as a support tool for a distribution company (DISTCO) to define a tariff structure that, while maximising its 
profit, satisfies the regulatory rules and gives guidelines to customers on how to choose the real-time tariff, that are sensitive to the price signals.

The paper is organised as follows: before introducing the optimisation model we will discuss the advantages of dynamic pricing and show briefly its superiority with respect to the static tariffs (section 2). Our dynamic pricing strategy is described then in section 3 . While the development of our model does not depend on any particular market structure, the regulatory rules have been formulated for the specific case of the recently deregulated Italian market. Section 4 will be thus devoted to the translation of the Italian profit ceiling constraints in mathematical terms to fit the dynamic pricing scheme. The definition of a robustness framework will be the subject of section 5 and then computational results will be presented in section 6 and some remarks will conclude the paper.

\section{Time Varying Pricing Methods}

Unlike other commodities, the pricing of electricity has been characterised for long time by a flat-rate tariff without obeying the offer-demand rule. This tariff structure clearly distorts reality. Generation cost varies substantially over the hours of the day, the weeks of the month and even the seasons of the year. The first improvements for the pricing strategies consists in applying static time varying tariffs such as time-of-use, critical peak pricing and demand reduction programs (Doucet and Kleit 2003). The main objective behind these methods is to offer some economic incentives for the consumers to decrease their demand during peak-load periods in order to avoid the use of high cost generation units. A further improvement is the use of real-time pricing in such a way that retail tariffs reflect the variations of the wholesale prices. In this way, the demand responsiveness could adjust faster to the price signals. It is to be noted that the application of real-time methods requires the availability of sophisticated measuring instruments and not just the traditional energy accumulating meters used for flatrate tariffs. In what follows we describe briefly these methods and we discuss their characteristics.

\subsection{Static Pricing Methods}

Pricing methods belonging to this category are called static because they are composed of price components that are known a priori even months before their application (Borenstein et al. 2002). Among these methods we cite:

- Time-of-use method (TOU): under this method prices vary in a pre-set way within certain blocks of time during the day. Three prices in general (called peak, shoulder, and offpeak prices) are used to capture the macroscopic shape of the wholesale prices. The main disadvantage of the TOU is related to the infrequency of prices adjustments and also to its inability to capture the price variation within the blocks.

- Critical peak pricing $(C P P)$ : it is based on a TOU scheme with an additional penalty price that applies only to some critical peak periods. It is up to the DISTCO to decide the periods in which the penalty is applied provided that the frequency of application should agreed by contract. Even though the CPP represents an improvement to the TOU it has the disadvantages that the penalty price is set in advance and that the number of occasions in which it can be applied is limited a priori.

- Demand reduction programs (DRP): under these programs the customer receives a reward following a reduction in demand. In other programs (better known as interruptible rates) the customer gives the right to the DISTCO to interrupt the service during some 
critical periods and receives a price discount in the other periods. These methods share, however, the precedent methods in their shortcomings.

It is also worthwhile noting that both the flat-rate tariffs and the static pricing methods have a common drawback of penalizing flexible retail customers who, despite their limited consumption during critical periods, are forced to pay the high rates caused by the customers with high demand during the peak systems, i.e. with substantial contribution to the high prices (Wolak 2001).

\subsection{Real-Time Pricing}

Tariffs based on Real-Time Pricing (RTP) do not charge preset components but apply different retail prices for different hours of the day and for the different days in order to be frequently aligned with wholesale prices. RTP programs have the advantages of increasing the demand responsiveness and of improving the market efficiency. Many experts, indeed, made clear declarations in favour of RTP. One of the supporters is Ray Gifford (chairman of the Colorado Public Utility Commission) who observed "if retail electricity prices reflected the cost of power, a demand-side response would bring the market back to equilibrium, dampening both high prices and volatility" (see Faruqui and George 2002). Severin Borenstein (director of University of California Energy Institute) argued that "electricity markets will suffer from chronic difficulties until end-users become more active participants" (reported in Bushnell and Mansur 2002). RTP has, indeed, many attractive features (Wolak 2001, Borenstein and Holland 2003, Doucet and Kleit 2003). For instance, RTP:

- encourages the demand side to be more active by forcing the DISTCOs to be more careful in their purchasing decisions;

- dampens price volatility and reduce the possibility of blackouts in case of generation shortage;

- avoids the inadequate investment in production capacity since demand responsiveness reduces demand at high price periods and thus makes unnecessary the installation of new peak generating units.

Nevertheless, these alleged advantages have been followed by just a few empirical studies. Most of them are based on the use of TOU estimates to predict the retail demand responsiveness to RTP signals (Faruqui and George 2002). According to an EPRI (Electrical Power Research Institute) report, a $2.5 \%$ reduction in California's electricity demand could reduce wholesale prices by $24 \%$ (reported in Johnson 2001). Yusta and Dominguez 2002 have presented a model to measure the industrial demand response to RTP that shows how the reduction of load can reach $38 \%$ during the most expensive hours compared with the TOU rate in the same hours. Wolak 2001b argued that the Californian system could count on 10000 MW capacity savings (the equivalent of 10 huge plants) during the summer peak periods by simply applying RTP strategies. Another significant evaluation reported in Johnson 2001 stated that according to a recent analysis, the United States could save $\$ 14.8$ billion annually through the implementation of real-time electricity pricing.

Despite these advantages, RTP has not been widely accepted or implemented. One reason has been the belief that small customers will not shift usage in response to price signals (Faruqui and George 2002). A second reason is that implementing RTP may not be costeffective in light of the incremental billing and sophisticated metering costs (Borenstein and Holland 2003). Indeed, Yusta and Dominguez 2002 observe that in the short term the 
reduction of the consumption in peak hours decreases the retail revenues and thus DISTCOs do not appear to have incentive to encourage RTP. However, they affirm that RTP can produce long term benefits derived from the delay of capacity investment.

\section{Dynamic Pricing Model}

Dynamic Pricing (DP) is a general term to indicate any electricity tariff that recognizes the inherent uncertainty in supply costs (Faruqui and George 2002). In the literature the DP term has been used to indicate RTP and sometimes even time-varying methods. The model we are proposing in this paper is composed of two different components: the first one is based on a TOU scheme and the second one follows the real-time wholesale price. Moreover, in order to be general, the model even provides a flat-rate tariff for customers who prefer time-invariant prices. Our pricing strategy is, thus, a hybrid tariff that takes into account the stochastic conditions of the system and consequently fits well the general term of DP. This approach is very attractive since we believe that it will preserve the advantages of the RTP discussed above and at the same avoids one of the main shortcomings of the static pricing methods, i.e. that of exposing retail customers to excessive prices volatility.

The problem to be solved by the DISTCO consists in maximising, over a time horizon $T$, its profits resulting as the difference between the revenues deriving from serving the customers and the costs of buying energy. The time horizon may be chosen to be several months (in Italy, for example, retail prices change every two months by regulation) but usually it is possible to consider shorter horizon without significant loss in the solution quality.

The model that we are proposing here is composed of two kinds of tariffs. The first one is a flat-rate tariff that does not change with time and with the market conditions. The second is a dynamic tariff that follows partially the wholesale market price. Any customer can choose the tariff that fits better its consumption behaviour and is also allowed to change tariff at any time.

We suppose that, for time period $t=1, \ldots, T$, the DISTCO buys part of the needed energy, say $Q^{t}$, by means of bilateral contracts at a unit price $P^{t}$ and the remaining required quantity, say $q_{l}^{t}$ corresponding to scenario $l$, from the wholesale market at the stochastic rate of $\lambda_{l}^{t}$. While $Q^{t}$ is set by means of a long-medium term energy planning of the DISTCO and will be thus considered as input data in our model, the stochastic quantities $q_{l}^{t}$, for $t=1, \ldots, T$ and $l=1, \ldots, L$ represent decision variables in our model.

The revenues derive from serving $N_{2}$ customers, say, $N_{1}$ of them are on flat-rate tariff and the remaining customers are on dynamic tariff. Each customer $i$ is characterised by his reserved capacity that we denote by $C_{i}$ for the flat tariff and by $C_{i}^{b}$ for the dynamic tariff, where $b$ represents the TOU block $(b=1,2$ or 3 for the three blocks: peak, shoulder, and offpeak prices). The reserved capacity, to be defined by contract, is the maximum instant capacity, expressed in $\mathrm{KW}$, that the customer is allowed to reach. In addition, each customer is characterised by his energy demand. We distinguish here, for each time period $t=1, \ldots, T$, between two demand quantities: a baseline deterministic demand $D_{i}^{t}$ to be agreed by contract with the customer $i$ and the random demand $d_{i, l}^{t}$ that represents the additional load required by customer $i$ under scenario $l$. In the following we will deal first with the case of nonnegative realizations $d_{i, l}^{t}$.

The idea of baseline load is a recently introduced concept to indicate the maximum quantity that a DP customer is allowed to consume without applying DP prices (Borenstein et 
al. 2002). In the context of RTP, in Wolak 2001b, for example, the author proposed to fix the baseline load to $85 \%$ of each hour usage from the last year the customer was on flat-rate tariff.

The flat-rate tariff is time-invariant and has three variables:

- $F P F$ (in $€$ ): fix price applied to each flat-rate customer during the time horizon $T$;

- $C P F$ (in $€ / \mathrm{KW}$ ): price of each capacity unit reserved to a flat-rate customer;

- $E P F$ (in $€ / \mathrm{KWh}$ ): price of each energy unit to be consumed by a flat-rate customer.

The overall cost for the flat-rate customer $i$ under any specific scenario $l$ can be expressed as follows:

$$
F C_{i, l}=F P F+C P F * C_{i}+E P F * \sum_{t \in T}\left(D_{i}^{t}+d_{i, l}^{t}\right)
$$

While flat-rate tariff customers will be charged the same energy price for both the baseline load and the random demand, the DP customers will pay the price corresponding of the TOU block for the baseline load and a real-time price for the random component. The resulting DP tariff has thus four set of variables:

- $F P D$ (in $€$ ): fix price applied to each DP customer during the time horizon $T$;

- $C P D^{b}$ (in €/KW): price of a capacity unit reserved to a DP customer during block $b$;

- $E P D^{b}$ (in $€ / \mathrm{KWh}$ ): TOU price of a baseline energy unit consumed by a DP customer during the TOU block $b$;

- $E P D_{l}^{t}$ (in $€ / \mathrm{KWh}$ ): real-time price of a random energy unit consumed by a DP customer under scenario $l$ during the time period $t$.

Similarly, the overall cost for the dynamic tariff customer $i$ under any specific scenario $l$ is the following:

$$
D C_{i, l}=F P D+\sum_{b=l}^{3}\left(C P D^{b} * C_{i}^{b} * \frac{N^{b}}{24}+\sum_{t \in T_{b}}\left(E P D^{b} * D_{i}^{t}+E P D_{l}^{t} * d_{i, l}^{t}\right)\right)
$$

As explained above the key of success of our strategy, in order to be accepted by the control market operator, is to limit the volatility risk for retail customers even though such restriction may introduce some increase in the TOU prices and a decrease in the objective function. This restriction is included into the model by limiting the variability between $E P D^{b}$ and $E P D_{l}^{t}$ to a DISTCO-defined parameter $u$, as will be explained in section 5 .

By referring to the stochastic programming terminology, our DP tariff consists in twostage decisions: the first-stage flat and TOU rates which are scenario-invariant and the second-stage (recourse action) energy rate that depends on the time period and on the observed scenario as well. The mathematical formulation of the DP model can be represented as follows: 
$\operatorname{Max} \Pi=\sum_{i=1}^{N_{1}}\left[F P F+C P F * C_{i}+E P F * \sum_{i=1}^{T}\left(D_{i}^{t}+\sum_{l=1}^{L} p_{l} d_{i, l}^{t}\right)\right]$

$$
\begin{aligned}
& +\sum_{i=N_{1}+1}^{N_{2}}\left[F P D+\sum_{b=1}^{3}\left(C P D^{b} \frac{N_{b}}{24} C_{i}^{b}+\sum_{t \in T_{b}}\left(E P D^{b} * D_{i}^{t}+\sum_{l=1}^{L} p_{l} E P D_{l}^{t} * d_{i, l}^{t}\right)\right)\right] \\
& -\sum_{t=1}^{T}\left[P^{t} Q^{t}+\sum_{l=1}^{L} p_{l} \lambda_{l}^{t} q_{l}^{t}\right]
\end{aligned}
$$

s.t.

$$
Q^{t}+q_{l}^{t}=\sum_{i=1}^{N_{2}}\left(D_{i}^{t}+d_{i, l}^{t}\right) \quad t=1, \ldots T ; l=1, \ldots L
$$

$\Pi \leq P C$

$$
-u \leq E P D^{b}-E P D_{l}^{t} \leq u \quad b=1,2,3 ; t=1, \ldots T_{b} ; l=1, \ldots L
$$

$E P F \geq \theta_{1} \frac{\sum_{t=1}^{T}\left(P^{t} Q^{t}+\sum_{l=1}^{L} p_{l} \lambda_{l}^{t} q_{l}^{t}\right)}{\sum_{t=1}^{T}\left(Q^{t}+\sum_{l=1}^{L} p_{l} q_{l}^{t}\right)}$

$E P D^{b} \geq \theta_{2}^{b} \frac{\sum_{t \in T_{b}}\left(P^{t} Q^{t}+\sum_{l=1}^{L} p_{l} \lambda_{l}^{t} q_{l}^{t}\right)}{\sum_{t \in T_{b}}\left(Q^{t}+\sum_{l=1}^{L} p_{l} q_{l}^{t}\right)} \quad b=1,2,3$

$0.5 * C P D^{b-1} \leq C P D^{b} \leq 0.8 * C P D^{b-1} \quad b=2,3$

$0.5 * E P D^{b-1} \leq E P D^{b} \leq 0.8 * E P D^{b-1} \quad b=2,3$

$F P F, C P F, E P F, F P D \geq 0$

$$
\begin{array}{ll}
C P D^{b}, E P D^{b} \geq 0 & b=1,2,3 \\
E P D_{l}^{t} \geq 0 & t=1, \ldots T ; l=1, \ldots L
\end{array}
$$

Constraints (2) ensure the supply-demand equilibrium at each time period and under whatever scenario observed. Constraint (3) imposes a profit ceiling $P C$ on the objective value $\Pi$ in order to respect eventual regulatory constraints aiming at protecting the consumers against volatile retail prices. In the next section we will describe an example of real profit ceiling application as ordered by the electricity Italian Authority. The set of constraints (4) limit the variability between first- and second-stage decisions. Constraint (5) ensures that the flat-rate energy component $\gamma_{1}$ will at least cover a fraction of the relative cost paid by the DISTCO. The coefficient $\theta_{1}$ in the right hand side is defined on the basis of the quantity of energy consumed by flat-rate customers. Similar parameters $\theta_{2}^{b}$ are used in the analogous constraints (6), referring to the three blocks of the TOU energy prices, to be applied to the DP customers. The choice of the parameters' values of $\theta_{1}$ and $\theta_{2}^{b}$ depends on the attitude of the 
DISTCO to move the cost coverage on the energy consumption tariff components rather than on the fixed components. This is clearly a strategic choice that depends on the DISTCO's managers and that influences directly the number of customers.

In (6) $T_{b}$ represents the set of time periods in which the block tariff $b$ is used and $N_{b}$ is its cardinality, i.e. the number of periods in which block $b$ applies. This last parameter is also used in the objective function in order to consider every capacity value $C_{i}^{b}$ with its effective weight. Constraints (7) and (8) impose an acceptable distinction between the blocks of the TOU prices on the basis of user-defined parameters that we set here to the values of 0.5 and 0.8. The remaining constraints impose non-negativity on all the decision variables.

Even though we have supposed till now that all the realizations of $d_{i, l}^{t}$ are nonnegative, the model can be generalized to include even negative values of $d_{i, l}^{t}$ provided some slight modifications. Indeed, the DISTCO may decide to promote energy savings behaviours by means of incentives consisting in allowing dynamic customers to have some realizations of the total energy that are less than their baseline demand (specially during peak-load periods). In this case the term $\sum_{l=l}^{L} p_{l} * E P D_{l}^{t} * d_{i, l}^{t}$ in the objective function will have a negative sign which represents a further advantage for the dynamic consumer. Such a policy will be also advantageous for the DISTCO as long as the savings deriving from not buying the corresponding quantity of energy in the wholesales market is bigger than the relative lack of profit. However, in this case it is reasonable to apply a discount price on negative realizations of demand that is a fraction, say $\xi$, of the dynamic energy price. In this way, the only modification to be reported to the model is limited to the objective function, and in particular in the term corresponding to the revenues from dynamic customers that becomes:

$$
\ldots+\sum_{i=N_{1}+1}^{N_{2}}\left[F P D+\sum_{b=1}^{3}\left(C P D^{b} \frac{N_{b}}{24} C_{i}^{b}+\sum_{t \in T_{b}}\left(E P D^{b} * D_{i}^{t}+\sum_{l=1}^{L} p_{l} \xi * E P D_{l}^{t} * d_{i, l}^{t}\right)\right)\right]-\ldots
$$

with the parameter $\xi$, to be chosen by the DISTCO, that equals to 1 for nonnegative realizations of the random demand and $0<\xi<1$ otherwise.

\section{Profit Ceiling Constraints}

The DP model has included a profit ceiling limitation represented in the general form (3). In this section we will discuss the profit ceiling constraint and give an example of its implementation for a real-life market.

From the economical point of view, profit ceiling constraints may be necessary as long as the market is not perfectly competitive. The perfect competition term in the electricity context is a complex issue that includes many conditions such as inexistence of market power and generators collusion, absence of supply shortage and entry barriers, developed retail competition, etc. The dissatisfaction of one of these conditions can represent a fatal risk leading to the failure of the electricity market. Actually, restructured markets worldwide are far to be perfectly competitive since the deregulation process in most of the countries is still either not completed or in an experimental phase.

Before describing the Italian regulatory constraints on retail tariffs it is worth mentioning that the introduction of profit ceiling constraints is not a recent issue but has been used even in the monopoly context before deregulation. A real example is reported by Sheen 
et al. 1994 who described the TOU pricing model applied by the Taiwan power company at the end of the eighties. The model includes a profit ceiling constraint formulated as a function of the installed capacity.

In the recently restructured Italian market, the electricity Authority has introduced, through the Order 204/99 (AEE 1999), a profit ceiling mechanism for the retail market (see also AEE 2001 and AEE 2004). According to these orders DISTCOs have to offer at least one basic tariff but are also allowed to propose additional special tariffs based, for example, on TOU or DP structure. The profit ceiling mechanism (more appropriately indicated in the Italian context as revenue cap) introduces two kinds of constraints: V1 to be applied to all the DISTCO's customers under any tariff structure and, in stochastic settings, under whatever scenario observed and V2 to be respected for just the basic tariff applied by the DISTCO ${ }^{1}$.

According to Order 204/99 constraint V1 limits the annual revenues that the DISTCO can have by serving all the customers belonging to the same consumption category. On the basis of the customer's voltage level the Italian Authority defines six different consumption categories. While V1 is a set of $L$ constraints, one for each scenario, for each consumption category, V2 applies to every single customer and there will be consequently as much constraints as the number of customers following any of the tariffs proposed by the DISTCO and again under whatever scenario that materializes.

In order to incorporate the regulatory constraints into our pricing model we assumed as basic tariff the flat-rate tariff and the DP scheme as a special tariff. In this case, constraint (3) will be replaced by $L$ constraint V1 of the form:

$$
\begin{aligned}
& \sum_{i=1}^{N_{1}}\left[F P F+C P F^{*} C_{i}+E P F * \sum_{t=1}^{T}\left(D_{i}^{t}+d_{i, l}^{t}\right)\right]+ \\
& \sum_{i=N_{1}+1}^{N_{2}}\left[F P D+\sum_{b=1}^{3}\left(C P D^{b} \frac{N_{b}}{24} C_{i}^{b}+E P D^{b} * \sum_{t \in T_{b}} D_{i}^{t}+\sum_{t \in T_{b}} E P D_{l}^{t} * d_{i, l}^{t}\right)\right] \\
& \leq\left[N_{2} \rho_{1}+\rho_{3} \sum_{i=1}^{N_{2}} \sum_{t=1}^{T}\left(D_{i}^{t}+d_{i, l}^{t}\right)\right] \quad l=1, \ldots, L
\end{aligned}
$$

by $L^{*} N_{1}$ constraints $\mathrm{V} 2$ to impose a maximum payment to be supported by a flat-rate customer (V2_F):

$$
\begin{aligned}
& F P F+C P F * C_{i}+E P F * \sum_{t=1}^{T}\left(D_{i}^{t}+d_{i, l}^{t}\right) \\
& \leq\left(\varepsilon_{1}+\varepsilon_{2} C_{i}+\varepsilon_{3} \sum_{t=1}^{T}\left(D_{i}^{t}+d_{i, l}^{t}\right)\right) \quad l=1, \ldots, L ; i=1, . ., N_{1}
\end{aligned}
$$

and by $L^{*}\left(N_{2}-N_{1}\right)$ constraints V2 to impose a maximum payment to be supported by a dynamic customer, whenever the flat rate tariff is applied for his consumption profile (considering that he always has the option to chooses to stay on flat tariff) (V2_D):

\footnotetext{
${ }^{1}$ The introduction of such constraints has represented a serious difficulty for most of the Italian DISTCOs who proposed tariff schemes that have been rejected by the Authority because they violated the revenue cap.
} 


$$
\begin{aligned}
F P F+\sum_{b=1}^{3} C P F \frac{N_{b}}{24} C_{i}^{b}+E P F^{*} \sum_{b=1}^{3} \sum_{t \in T_{b}}\left(D_{i}^{t}+d_{i, l}^{t}\right) \leq & \\
\left(\varepsilon_{1}+\varepsilon_{2} \sum_{b=1}^{3} \frac{N_{b}}{24} C_{i}^{b}+\varepsilon_{3} \sum_{b=1}^{3} \sum_{t \in T_{b}}\left(D_{i}^{t}+d_{i, l}^{t}\right)\right) & l=1, \ldots, L ; i=N_{1}+1, . ., N_{2}
\end{aligned}
$$

where all the parameters defined on the right hand side of all the expressions are Authority defined coefficients that depend on the customer's category and are frequently updated to capture the energy cost variations.

We should note how in both the sets of constraints V2_F and V2_D the same variables and parameters are used for both flat and dynamic customers. This is due to the fact that the regulator ensures a cap only on the basic tariff to leave the customers who choose other tariffs on their own risk. These constraints do not affect the dynamic tariff's components but just the flat tariff's ones, taking into account the possibility that also dynamic customers could choose this kind of tariff.

\section{Robustness Framework}

The formulation proposed above is a two-stage stochastic linear model including a set of constraints that limit the variability between the first- and second-stage decisions. The resulting problem belongs to the family of stochastic linear programs with limited recourse and can take advantage from the modelling approach SLP_LR proposed in Beraldi et al. 2003. This is a specialised framework that supports the decision maker in determining the acceptable dispersion limitation parameter $u$. Starting from the solution of the classical twostage model, i.e. without recourse limitation, the variability between the two stage decisions is then reduced progressively until the limitation becomes too aggressive and the corresponding problem results to be infeasible.

In order to apply this approach to our pricing model, we need to report a slight modification to the SLP_LR framework by imposing the variability limitation on a subset of the decision variables. Indeed, instead of the problem setting considered in Beraldi et al. 2003 in which all the decision components are subject to restriction, in our case only the energy components of the DP tariff should respect the variability limitation. This can be carried out by using a recursive approach whose iterations consist in updating the parameter $u$ as follows

$$
u=\max _{\substack{l=1, \ldots, L \\ b=1,2,3 \\ t \in T_{b}}}\left|E P F^{b}-E P D_{l}{ }_{l}^{t}\right|-\min _{\substack{l=1, \ldots, L \\ b=1,2,3 \\ t \in T_{b}}}\left|E P F^{b}-E P D_{l}{ }_{l}^{t}\right|
$$

in order to enforce tighter limiting conditions on the two stage variables. The iterative procedure can be summarized as following:

\section{Limited Recourse Procedure}

/* solve the classical two-stage problem without any variability limitation */ solve_problem (solution, is_feasible, limitation_level);

while (is_feasible) do

/* update $u$ according to the expression (15) */

update_u ();

/* solve the limited recourse problem with the new value of $u *$ /

solve_problem (solution, is_feasible, limitation_level);

end do 
As a further consequence to the above modifications we should adapt the variability measure in order to suit our model specification:

$$
\rho=\sum_{l=1}^{L} p_{l}\left\|E P D_{l}^{t}-E P F^{b}\right\| \quad \text { for } t \in T_{b} .
$$

This measures will be used in the sequel of the paper to analyze the model behaviour. It represents the mean distance of the recourse decisions from the first-stage ones.

The limited recourse iterative approach represents a relevant advantage to the DISTCO since it allows to find the acceptable trade-off between the variability limit and the (possible) resulting decrease in the objective function. Moreover, the adoption of this framework can be motivated by the opportunity for the DISTCO to define several dynamic tariff schemes by choosing different values of variability parameter $u$, that, however, represent different attitudes to risk. In this way, the DISTCO could take a competitive advantage by offering to the customers a very flexible range of tariff solutions.

\section{Computational Results}

The dynamic pricing model has been tested on several instances of a test problem generated by simulating input data representing some realistic scenarios of the Italian market. The number of customers chosen refers to a rather small DISTCO with 300 customers 200 of which are on flat-rate tariff and the others are on the dynamic tariff. The required input data have been extracted from the data available on the web site of the Italian market and grid operators and the Authority ${ }^{2}$ and refer to the month of June 2004. On the basis of these data 10 different scenarios with the same likelihood of occurrence have been generated to model the random clearing price and the dynamic demands by using the bootstrapping technique (Efron and Tibshirani 1993). The quantity of energy purchased by bilateral contracts $Q^{t}$ has been set to a value close to the sum of the baseline energy of all the customers. All the parameters present in the right hand side of the profit ceiling constraints are Authority defined values that depend on the category of the consumers in relation to the consumption voltage (AEE 2004). In our experiments we have considered the following parameters defined for the category of non-residential users with low voltage: $\rho_{1}(€ /$ year $)=176.36 ; \rho_{3}(€ / \mathrm{kWh})=0.015$; $\varepsilon_{1}(€ /$ year $)=19.26 ; \varepsilon_{2}(€ / \mathrm{kW}$ year $)=30.77 ; \varepsilon_{3}(€ / \mathrm{kWh})=0.001$.

The test problem considered here is specific to 300 customers belonging to the category of non-residential users with low voltage but other test problems for other categories have been considered showing the same behaviour of the results presented below. The time horizon has been fixed to one day divided into 24 intervals and the number of four blocks of the TOU tariff has been assumed in order to meet the Italian regulations. The resulting problem has a dual block-angular structure with 252 variables, 3501 constraints and 12895 non-zeros.

Several instances of our test problem have been solved by varying the limited recourse parameter $u$. We also solved the unconstrained version of the problem, i.e. without any limitation on the variability between the first-and the second-stage decisions (that we will improperly call unconstrained model).

Our experiments have also included the case in which upper and lower bounds are imposed on the decision variables. Imposing such bounds is usual in any industrial pricing process in order to express the preferences of the marketing manager. Several appropriate

\footnotetext{
${ }^{2}$ http://www.autorita.energia.it
} 
bounds that are compatible with the Italian market's specifications have been tested. In all the cases a similar behaviour has been observed confirming the robustness of our model.

Table 1: Flat-rate and TOU tariff

\begin{tabular}{|l|c|c|c|c|}
\cline { 2 - 5 } \multicolumn{1}{c|}{} & \multicolumn{5}{c|}{ Block } \\
\hline Variable & $\mathbf{1}$ & $\mathbf{2}$ & $\mathbf{3}$ & $\mathbf{4}$ \\
\hline$F P F(€)$ & \multicolumn{5}{c|}{0.30} \\
\hline$C P F(€ / \mathrm{kW})$ & \multicolumn{5}{c|}{0.082} \\
\hline$E P F(€ / \mathrm{kWh})$ & \multicolumn{5}{c|}{0.03} \\
\hline$F P D(€)$ & 0.05 & 0.04 & 0.032 & 0.026 \\
\hline$C P D^{b}(€ / \mathrm{kW})$ & 0.1 & 0.068 & 0.055 & 0.044 \\
\hline$E P D^{b}(€ / \mathrm{kWh})$ & &
\end{tabular}

Our emphasis here is twofold: from one side testing the validity of the dynamic pricing model and from the other side showing the importance of the limited recourse technique in defining an efficient two-stage tariff scheme. In order to discuss the validity of the model we report in Tables 1 and 2 an example of a resulting tariff for the particular value of $u=20 \%$. More specifically, Table 1 reports the values of the flat-rate and those of the TOU variables (block 1 refers to the most loaded periods and block 4 for the less loaded periods) and Table 2 reports the real-time variables for the 10 defined scenarios and one representative time period for each block (only slight variation is observed among the periods of the same block). These results show that the model's output offers reasonable flat and dynamic tariff structures that are comparable with real tariffs actually applied in the Italian market (a commercial tariff example is reported in EAM 2004). Moreover, we can note some interesting features in the proposed tariff. First the model "penalizes" the flat customers by a tariff having a higher fix rate with respect to the corresponding TOU component. This can be seen as a risk aversion behaviour of the DISTCO in front of the demand inelasticity of flat customers. Second, it is worth noting in Table 2 how the real-time price varies for the different scenarios in the loaded periods and tends to stabilize in off-peak periods. This is reasonable since the real-time tariff component should follow the profile of the clearing price that increases remarkably only during the peak periods.

Table 2: Real-time tariff (in $€ / \mathrm{kWh}$ )

\begin{tabular}{|l|c|c|c|c|}
\cline { 2 - 5 } \multicolumn{1}{c|}{} & \multicolumn{4}{c|}{ Block } \\
\hline Scenario & $\mathbf{1}$ & $\mathbf{2}$ & $\mathbf{3}$ & $\mathbf{4}$ \\
\hline 1 & 0.087 & 0.055 & 0.044 & 0.035 \\
\hline 2 & 0.1 & 0.055 & 0.044 & 0.035 \\
\hline 3 & 0.1 & 0.055 & 0.044 & 0.035 \\
\hline 4 & 0.1 & 0.055 & 0.044 & 0.035 \\
\hline 5 & 0.099 & 0.055 & 0.044 & 0.035 \\
\hline 6 & 0.08 & 0.071 & 0.044 & 0.035 \\
\hline 7 & 0.096 & 0.055 & 0.044 & 0.035 \\
\hline 8 & 0.094 & 0.055 & 0.044 & 0.035 \\
\hline 9 & 0.08 & 0.055 & 0.044 & 0.035 \\
\hline 10 & 0.08 & 0.055 & 0.044 & 0.035 \\
\hline
\end{tabular}


The effect of the limited recourse is discussed here in terms of variation of both the objective function and the variability measure $\rho$ with the parameter $u$ (we consider here the values $u=5 \%, 10 \%, 20 \%$ and 30\%). The results are reported in Table 3 . In our case, while the value of $\rho$ increases with the $u$, as depicted in Figure 2, the objective function remains unaltered for any value of $u$ and for the unconstrained case as well. This is due to the achievement of the maximum profits imposed by the profit ceiling constraints. Indeed, by eliminating the profit ceiling constraints the objective value observes a different behaviour since it takes higher values with respect to the previous case and it also varies with the parameter $u$, as shown in Figure 3.

Table 3: Effect of limited recourse

\begin{tabular}{lrrrr}
\cline { 2 - 5 } & \multicolumn{2}{c}{ With profit ceiling } & \multicolumn{2}{c}{ Without profit ceiling } \\
\hline $\mathbf{u}(\boldsymbol{\%})$ & Objective value & Mean distance & Objective value & Mean distance \\
\hline 0 & 38874,3 & 15,34 & 91167,85 & 15,34 \\
5 & 38874,3 & 1,66 & 87264,33 & 1,38 \\
10 & 38874,3 & 3,40 & 87744,33 & 2,76 \\
20 & 38874,3 & 6,80 & 88704,34 & 5,52 \\
30 & 38874,3 & 10,71 & 89342,34 & 7,39 \\
\hline
\end{tabular}

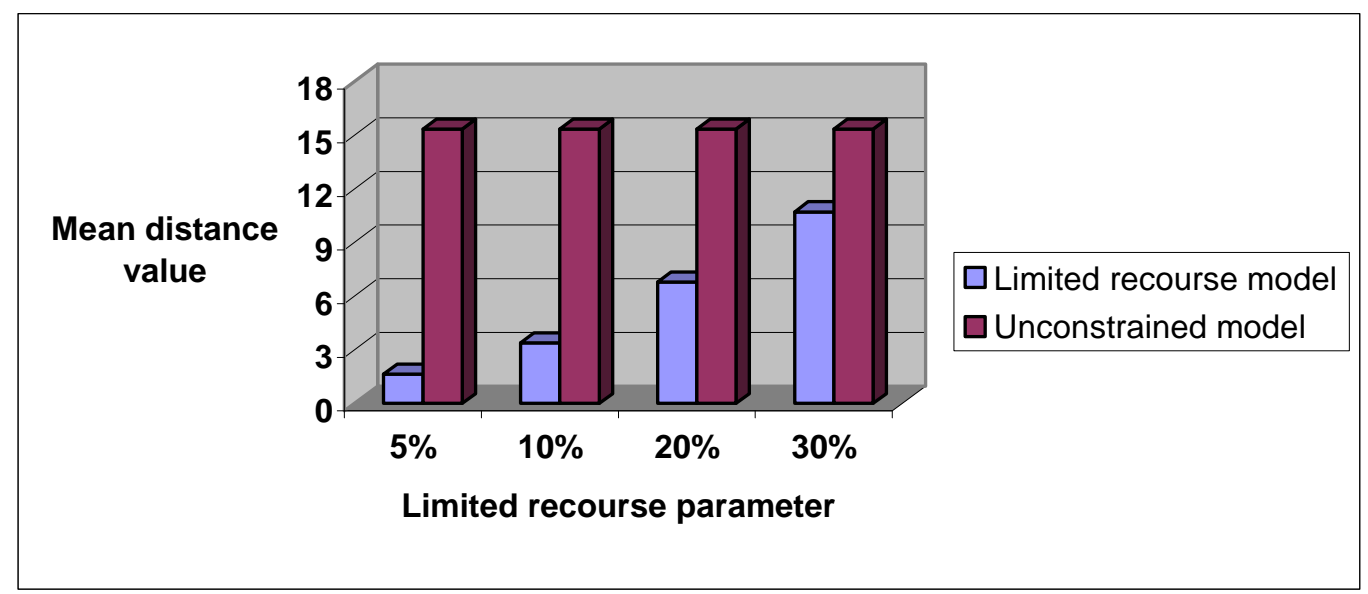

Figure 2: Mean distance values vs. limited recourse parameter (with profit ceiling constraints)

The defined structure has a particular attractive feature that offers a real advantage for the DISTCO. It can offer a dynamic tariff having whatever value of the parameter $u$ without causing any reduction in its profits. This means that any DP customer can choose whatever variability value $u$ which will produce different values of the first- and second-stage decisions but without influencing the DISTCO's profits.

\section{Concluding Remarks}

With the deregulation of the electricity markets many new optimisation models and solution algorithms are to be developed. This paper proposes the use of stochastic programming to define a retail tariff structure in the context of the open market. The structure offers a flat-rate tariff but also proposes an attractive DP scheme that contributes to the efficiency of the market by including a component that depends on the wholesales price of the energy. The DP 
tariff charges, in a first-stage, a TOU rate for a baseline demand and then makes a recourse action by applying a real-time stochastic price for any additional energy consumed. To avoid remarkable real-time price volatility the model includes variability constraints between the two stages decisions. In the paper we formulated also profit cap constraints as proposed by the recently restructured Italian market and we discussed the model's validity and the effect of the limited recourse approach by solving some instances of a realistic test problem.

Future research in this field can be developed along a variety of directions. A particular attention may be given to the study of the effect of dynamic tariffs on the behaviour of the customers and the way they are forced to become responsive. Moreover, the introduction of a risk aversion component into the model may make it more realistic and effective for real word applications. Another possibility is to extend the dynamic model to other tariff structures such as those based on the binomial rates. Another useful development may be the application of our model to solve real problems referring to a large DISTCO by (i) collecting firm-specific data, (ii) considering a longer time horizon, and (iii) performing a more sophisticated and realistic scenarios generation analysis. These extensions should be computationally intensive since they introduce a huge number of additional variables and constraints and the limited recourse algorithm applied here should be unable to deal with such complexity. The development of specialized algorithms based on a combination of row and column generation methods, combined with some feasible geographical splitting, could be a key issue to explore in this context. Moreover, it will be necessary to include some of the advanced techniques, typically used in stochastic programming, to limit the number of scenarios such as the scenario reduction algorithm or the constraint aggregation principle.

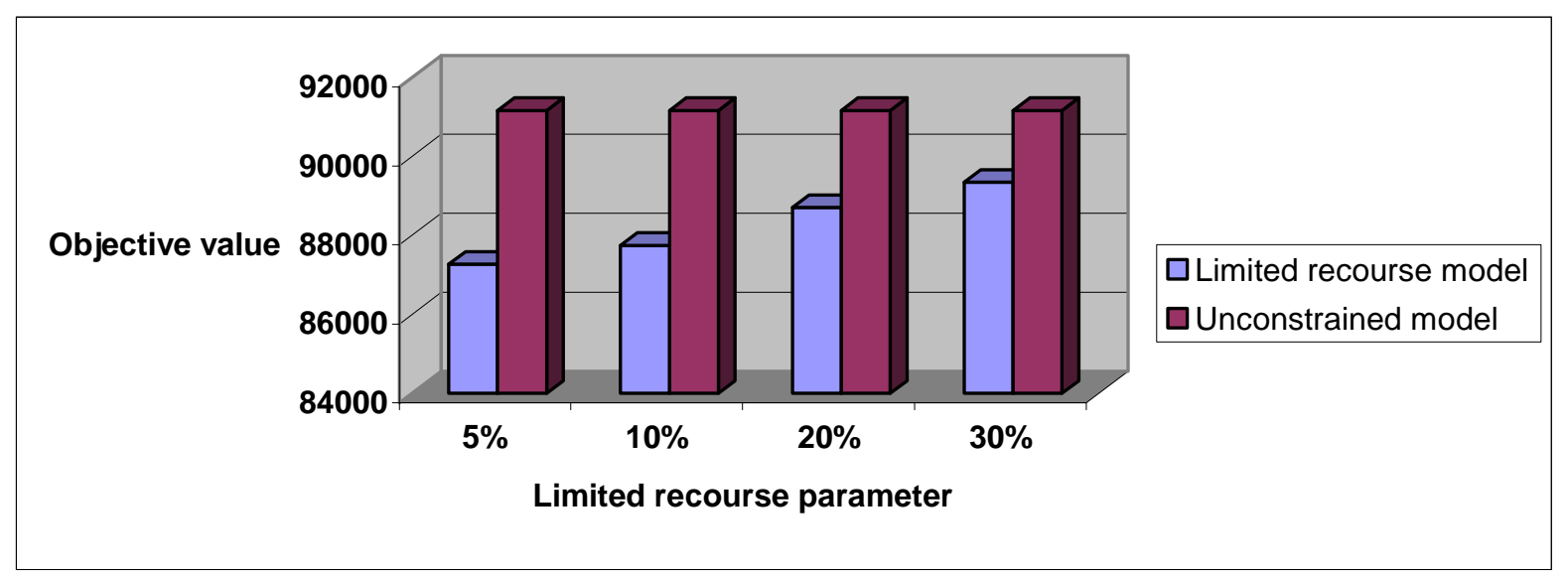

Figure 3: Objective function vs. limited recourse parameter (without profit ceiling constraints) 


\section{References}

Authority for Electric Energy and Gas (1999) Delibera 204/99. Available at http://www.autorita.energia.it/docs/index.htm.

Authority for Electric Energy and Gas (2001) Delibera 228/01. Available at http://www.autorita.energia.it/docs/index.htm.

Authority for Electric Energy and Gas (2004) Testo integrato delle disposizioni dell'autorità per l'energia elettrica e il gas per l'erogazione dei servizi di trasmissione, distribuzione, misura e vendita dell'energia elettrica. Periodo di regolazione 2004-2007. Delibera 5/04. Available at http://www.autorita.energia.it/docs/index.htm.

Beraldi P, Musmanno R, Triki C, Zenios SA (2003) Limited Recourse in Two-Stage Stochastic Linear Programs. Journal of Information and Optimization Science 24(3):445-65.

Borenstein S (2004) The Long-Run Effects of Real-Time Electricity Pricing. Energy Journal, forthcoming.

Borenstein S, Holland SP (2003) Investment Efficiency in Competitive Electricity Markets with and without Time-Varying Retail Prices. CSEM WP 106R. University of California Energy Institute. Available at http://paleale.eecs.berkeley.edu/ucei/pubscsemwp.html.

Borenstein S, Jaske M, Rosenfeld A (2002) Dynamic Pricing, Advanced Metering and Demand Response in Electricity Markets. CSEM WP 105. University of California Energy Institute. Available at http://paleale.eecs.berkeley.edu/ucei/pubs-csemwp.html.

Bushnell J, Mansur E (2002) The Impact of Retail Rate Deregulation on Electricity Consumption in San Diego. PWP 82. University of California Energy Institute.Available at http://www.ucan.org/law_policy/energydocs/bushnelldsm.pdf .

Doucet JA, Kleit A (2003) Metering in Electricity Markets: When is More Better? In: Crew MA, Schuh JC (eds) Markets, Pricing, and Deregulation of Utilities. Kluwer Academic Publisher, 87:108.

EAM (2004) Opzioni tariffarie di trasporto e vendita di energia elettrica per clienti allacciati in bassa tensione, usi diversi dall'abitazione (clienti vincolati). Available at http://www.miservi.it/miserviWeb/serviziAEM/pmi/informativa/tariffe_ele_pmi.jsp.

EFFLOCOM (Energy EFFiciency and LOad curve impacts of COMmercial development in competitive markets) project reports. Available at www.efflocom.com.

Efron B, Tibshirani RJ (1993) An Introduction to the Bootstrap. Chapman \& Hall/CRC.

Faruqui A, George SS (2002) The Value of Dynamic Pricing in Mass Markets. Elsevier Science Inc. Available at http://www.dramcoalition.org/id57.htm.

Johnson RR (2001) Residential Meters: Adoption at Last?. Public Utility Fortnightly Supplement: Fortnightly's Energy Customer Management Fall 2001:58-63.

Pettersen E (2004) Managing End-users Flexibility in Electricity Markets. PhD thesis, NTNU, Trondheim, Norway.

Sheen JN, Chen CS, Yang JK (1994) Time-Of-Use Pricing for Load Management Programs in Taiwan Power Company. IEEE Transaction on Power Systems 9(1):388-96.

Wolak FA (2001) Designing a Competitive Wholesale Market that Benefits Consumers. Department of Economics, Stanford University. Available at http://www.stanford.edu/ wolak/.

Wolak FA (2001b) Want 10.000 megawatts now? Use variable power pricing. San Jose Mercury News 4 May 2001. Available at http://www.stanford.edu/ wolak/.

Yusta JM, Dominguez JA (2002) Measuring and Modelling of Industrial Demand Response to Alternative Prices of the Electricity. Proceedings of the $14^{\text {th }}$ PSCC in Sevilla. 